\title{
Accuracy of Bioelectrical Impedance Consumer Devices for Measurement of Body Composition in Comparison to Whole Body Magnetic Resonance Imaging and Dual X-Ray Absorptiometry
}

\author{
Anja Bosy-Westphal ${ }^{\mathrm{a}}$ Wiebke Later ${ }^{\mathrm{a}}$ Britta Hitze $^{\mathrm{a}}$ Tetsuya Sato $^{\mathrm{b}}$ Elke Kossel $^{\mathrm{c}}$ \\ Claus-Christian Glüer ${ }^{c}$ Martin Heller ${ }^{c}$ Manfred James Müller ${ }^{a}$ \\ a Institut für Humanernährung und Lebensmittelkunde, Christian-Albrechts-Universität, Kiel, Germany \\ b Omron Healthcare Co., Ltd, Kyoto, Japan \\ ${ }^{c}$ Klinik für Diagnostische Radiologie, Universitätsklinikum Schleswig-Holstein, Campus Kiel, Germany
}

\section{Key Words}

Body composition - Bioelectrical impedance analysis . Accuracy · Magnetic resonance imaging · Dual-energy X-ray absorptiometry

\section{Summary}

Objective: To compare body composition determined by bioelectrical impedance (BIA) consumer devices against criterion estimates determined by whole body magnetic resonance imaging (MRI) and dual energy $\mathrm{X}$-ray absorptiometry (DXA) in healthy normal weight, overweight and obese adults. Methods: In 106 adults (54 females, 52 males, age $54.2 \pm 16.1$ years, BMI $\left.25.8 \pm 4.4 \mathrm{~kg} / \mathrm{m}^{2}\right)$ fat mass $(\mathrm{FM})$, skeletal muscle mass (SM), total body bone-free lean mass (TBBLM), and level of visceral fat mass (VF) were estimated by 3 single-frequency bipedal (foot-to-foot) and one tretrapolar BIA device, and compared to body composition measured by MRI and DXA. Bland-Altman and simple linear regression analyses were used to determine agreement between methods. Results: \%FM $M_{D X A}, S_{M R I}$ or TBBLM $M_{D X A}$ showed good relative and absolute agreement with two bipolar and one tetrapolar instrument $\left(r^{2}=0.92-0.96\right.$; all $\mathrm{p}<0.001$; mean bias $<1.5 \% \mathrm{FM}$ and $<1 \mathrm{~kg}$ SM or TBBLM) and less relative and absolute agreement for another bipolar device $\left(r^{2}=0.82\right.$ and 0.84 , mean bias $\sim 3 \% \mathrm{FM}$ and $\sim 3 \mathrm{~kg}$ $\mathrm{SM})$. The $95 \%$ limits of agreement (bias \pm 2 SD) were narrowest for the tetrapolar device (-6.59 to $4.61 \% \mathrm{FM}$ and -4.62 to $4.74 \mathrm{~kg} \mathrm{SM}$ ) and widest for bipolar instruments (up to -14.54 to $8.58 \% \mathrm{FM}$ and -9.52 to $3.92 \mathrm{~kg} \mathrm{SM}$ ). Systematic biases for \%FM were found for all bipedal devices, but not for the tetrapolar instrument. Conclusion: Because of the lower agreement between foot-to-foot BIA and DXA or MRI for the assessment of body composition in individuals, tetrapolar electrode arrangement should be preferred for individual or public use. Bipolar devices provide accurate results for field studies with group estimation.
Introduction

There is an increasing demand for body composition analysis in personal use or homecare to monitor weight status, weight loss therapy, or outcome of strength or endurance exercise. Bioelectrical impedance analysis (BIA) consumer devices are cheap and easy to use for noninvasive indirect assessment of body composition and offer a variety of outcome parameters (e.g. total body water, fat mass (FM), fat free mass, muscle mass). In addition, some BIA devices intend to predict visceral FM (as an estimate of cardiometabolic risk) and resting energy expenditure (REE; which may be used to estimate energy requirement for weight loss /gain or weight maintenance). The investigation of the accuracy of single-frequency BIA provided inconsistent results, with some studies showing a good accuracy [1-5] and others reporting only a poor agreement between BIA and reference methods [6-8]. The reasons for these discrepancies may be at least threefold. First, because BIA uses a statistical relationship between electrical properties of tissues and the target variable, it can be referred to as a prediction technique in contrast to reference methods like air displacement plethysmography, dual energy X-ray absorptiometry (DXA) or magnetic resonance imaging (MRI) that measure body compartments [9, 10]. Therefore, BIA equations are population-specific, and the accuracy of BIA results is considerably dependent on the agreement of physical characteristics, weight status, ethnicity and age between the subject and the reference population used to generate the BIA algorithm [11-13].

Second, the market offers a wide range of different BIA consumer devices. Differences between impedance systems are also known to influence the validity of BIA results [14]. Bipolar foot-to-foot devices that require the subject only standing

\section{KARGER}

Fax +497614520714

Information@Karger.de

www.karger.com (c) 2008 S. Karger GmbH, Freiburg

Accessible online at:

www.karger.com/ofa 
bare-footed on surface electrodes can be distinguished from tetrapolar devices that include hand electrodes as well. In the former type, impedance is measured through the legs and lower trunk only, whereas in the latter type impedance measurements also include the arms and upper trunk. In addition, differences in the shape, conductivity and arrangement of electrodes may lead to systematic bias between methods and require device-specific algorithms.

Third, a constant hydration of lean mass is an underlying assumption of the two-compartment (2C) model of body composition analysis. However, this assumption is violated under clinical conditions like renal or heart failure or severe obesity (i.e. higher hydration of lean mass) as well as under unstable or non steady-state conditions (e.g. weight loss) [15]. Although this argument confers a limitation to all $2 \mathrm{C}$ methods of body composition analysis, even to reference methods like densitometry or DXA, multifrequency BIA or bioelectrical impedance spectroscopy (BIS) may be more valid under these conditions $[1,16,17]$.

The aim of the present study was to analyze the validity of four different single-frequency BIA consumer devices using MRI and DXA as criterion methods. A Caucasian population of 106 men and women with a wide age and BMI range (22-78 years, $16.8-40.1 \mathrm{~kg} / \mathrm{m}^{2}$ ) was investigated.

\section{Subjects and Methods}

\section{Participants}

The study group was recruited by local advertisement and consisted of 106 healthy euthyroid and weight-stable subjects (nonpregnant or lactating) aged 22 to 78 years with a BMI range of $16.8-40.2 \mathrm{~kg} / \mathrm{m}^{2}$. Exclusion criteria were smoking and any medication influencing energy expenditure or body composition (e.g. $\beta$-blockers or diuretics). The study protocol was approved by the local ethical committee of the Christian-Albrechts-University Kiel. Each participant provided informed written consent prior to participation

Subjects arrived in the morning after an overnight fast at the Institute for Human Nutrition and Food Science of the Christian-Albrechts-University, Kiel. REE was measured at the metabolic ward of the institute followed by body composition analysis with BIA and afterwards DXA. Whole body MRI was done after lunch in the afternoon of the same day. Both DXA and MRI took place at the Clinic for Diagnostic Radiology, Universitätsklinikum Schleswig-Holstein. Body height was measured to the nearest $0.5 \mathrm{~cm}$ with a stadiometer (Seca, Hamburg, Germany).

\section{Bioelectrical Impedance Analysis}

Body composition was assessed by single-frequency BIA devices using the Tanita Inner Scan Model BC-532 (Tanita Europe GmbH., Sindelfingen, Germany), Soehnle Body Balance (Soehnle-Waagen GmbH and Co. KG, Murrhardt, Germany), Omron BF-400, and Omron BF-500 (Omron Medizintechnik, Mannheim, Germany). Manufacturers' equations were used to predict \%FM (all scales), skeletal muscle mass ( $\mathrm{SM}$, in $\mathrm{kg}$ ) (Omron BF-500 and Soehnle Body Balance), total body bone-free lean mass (TBBLM, in kg) (Tanita Inner Scan BC-532), grade of visceral fat (VFlevel) (Omron BF-500 and Tanita Inner Scan BC-532) and REE (Omron BF-500 and Tanita Inner Scan BC-532). Subjects were measured barefoot in light underwear following manufacturer's instructions. The Omron HBF-500 BIA device uses eight electrodes in a tetrapolar
Table 1. Physical characteristics, body composition and resting energy expenditure (REE) of the study population (data are means \pm SD)

\begin{tabular}{llll}
\hline & Females $(\mathrm{n}=54)$ & Males $(\mathrm{n}=52)$ & All $(\mathrm{n}=106)$ \\
\hline Age, years & $43.5 \pm 16.1$ & $46.9 \pm 16.0$ & $45.2 \pm 16.1$ \\
Height, $\mathrm{m}$ & $1.67 \pm 0.05$ & $1.77 \pm 0.05^{*}$ & $1.72 \pm 0.07$ \\
Weight, $\mathrm{kg}$ & $71.65 \pm 13.61$ & $81.11 \pm 12.25^{*}$ & $76.29 \pm 13.75$ \\
BMI, kg/m & $25.8 \pm 4.9$ & $25.9 \pm 3.9$ & $25.8 \pm 4.4$ \\
\hline FM $_{\text {DXA }} \%$ & $33.74 \pm 8.47$ & $20.23 \pm 6.18^{*}$ & $27.11 \pm 10.04$ \\
TBBLM $_{\text {DXA }}, \mathrm{kg}$ & $43.03 \pm 4.51$ & $59.15 \pm 6.59$ & $50.94 \pm 9.85$ \\
SM $_{\text {MRI }}, \mathrm{kg}$ & $19.87 \pm 3.20$ & $29.15 \pm 4.65^{*}$ & $24.42 \pm 6.12$ \\
VF $_{\text {MRI }}$, level & $6.32 \pm 3.00$ & $9.76 \pm 5.09^{*}$ & $8.04 \pm 4.50$ \\
\hline REE $_{\text {measured, }}$ kcal/day & $1,421 \pm 157$ & $1,733 \pm 210^{*}$ & $1,574 \pm 242$ \\
\hline
\end{tabular}

*Significant difference between sexes $(\mathrm{p}<0.001)$.

Table 2. Pearson correlation coefficients between parameters assessed by different BIA devices and reference methods

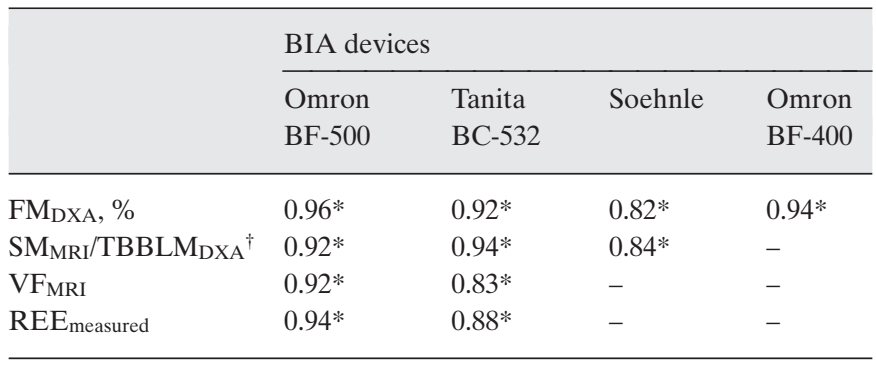

TBBLM according to Tanita device was compared with TBBLM from DXA.

* $\mathrm{p}<0.001$.

arrangement that requires the subject standing on metal footpads in bare feet and grasping a pair of electrodes fixed on a handle with arms extended in front of the chest. The other BIA devices were bipolar foot-to-foot instruments. Coefficient of repeated measurements (CVintra) for FM was $4-6 \%$.

\section{Dual-Energy X-Ray Absorptiometry}

Whole body measurement was carried out using a QDR4500A (Hologic Inc., Bedford, MA, USA). Scans were performed by a licensed radiological technician. Before measurement, calibration was done with a phantom. During the 5-min scan, subjects lay supine with arms and legs at their sides. Manufacturer's software (version V8.26a:3) was used for analysis of FM and TBBLM. The precision of FM measurements by DXA has been reported to be $2-3 \%$ [18].

\section{Magnetic Resonance Imaging}

Volumes of SM, and area of visceral fat (VF) were assessed by MRI using a Magnetom Avanto 1.5-T scanner (Siemens Medical Systems, Erlangen, Germany). Subjects were examined in a supine position with their arms extended above their heads. Continuous transversal images with $10 \mathrm{~mm}$ slice thickness and no interslice gaps were obtained from wrist to ankle using a T1-weighted gradient echo sequence (TR $575 \mathrm{~ms}$, TE $15 \mathrm{~ms}$ ). Images in abdominal and thoracic regions were obtained with the subjects holding their breath. Every second image (interval of $20 \mathrm{~mm}$ for analysis) was segmented manually and analyzed using ZedView $3.1^{\mathrm{TM}}$ software 
Table 3. Results of the limits of agreement analysis: mean result $( \pm \mathrm{SD})$, bias and $95 \%$ limits of agreement $( \pm 2 \mathrm{SD})$ for fat mass $(\mathrm{FM})$, skeletal muscle mass (SM), visceral fat level $\left(\mathrm{VF}_{\text {level }}\right)$ and resting energy expenditure (REE) measured by criterion method and compared with prediction by BIA

\begin{tabular}{|c|c|c|c|c|}
\hline & \multicolumn{4}{|l|}{ BIA devices } \\
\hline & Omron BF-500 & Tanita BC-532 & Soehnle & Omron BF-400 \\
\hline $\begin{array}{l}\mathrm{FM}_{\mathrm{BIA}}, \% \\
\Delta \mathrm{FM}_{\mathrm{DXA}-\mathrm{BIA}}, \%^{\mathrm{a}} \\
\text { Correlation }^{b}, r\end{array}$ & $\begin{array}{l}28.10 \pm 9.62 \\
-0.99 \pm 5.60 * * \\
0.15\end{array}$ & $\begin{array}{l}28.53 \pm 8.97 \\
-1.37 \pm 7.96^{* *} \\
0.30^{* *}\end{array}$ & $\begin{array}{l}30.14 \pm 9.01 \\
-2.98 \pm 11.56^{* *} \\
0.20^{*}\end{array}$ & $\begin{array}{l}27.59 \pm 9.27 \\
-0.48 \pm 6.62 \\
0.24^{*}\end{array}$ \\
\hline $\begin{array}{l}\text { SMBIA }, \mathrm{kg} / \mathrm{TBBLM}_{\text {BIA }}, \mathrm{kg} \\
\Delta \text { SMMRI-BIA }^{\mathrm{a}}, \mathrm{kg} \\
\Delta \text { TBBLM }_{\text {DXA-BIA }}{ }^{\mathrm{a}}, \mathrm{kg} \\
\text { Correlation }^{b}, r\end{array}$ & $\begin{array}{l}24.54 \pm 5.63 \\
0.06 \pm 4.68 \\
- \\
0.22 *\end{array}$ & $\begin{array}{l}51.64 \pm 9.30 \\
- \\
-0.79 \pm 6.64 * \\
0.18\end{array}$ & $\begin{array}{l}27.31 \pm 5.61- \\
-2.80 \pm 6.72 * * \\
- \\
0.18\end{array}$ & $\begin{array}{l}- \\
- \\
-\end{array}$ \\
\hline $\begin{array}{l}\mathrm{VF}_{\mathrm{BIA}}, \text { level } \\
\Delta \mathrm{VF}_{\mathrm{MRI}-\mathrm{BIA}}{ }^{\mathrm{a}} \text {, level } \\
\text { Correlation }^{b}, r\end{array}$ & $\begin{array}{l}7.85 \pm 3.83 \\
0.19 \pm 3.62 \\
0.38^{* * *}\end{array}$ & $\begin{array}{l}7.96 \pm 4.73 \\
0.27 \pm 5.18 \\
-0.02\end{array}$ & $\begin{array}{l}- \\
- \\
-\end{array}$ & $\begin{array}{l}- \\
- \\
-\end{array}$ \\
\hline $\begin{array}{l}\mathrm{REE}_{\mathrm{BIA}}, \mathrm{kcal} / \mathrm{day} \\
\Delta \mathrm{REE}_{\text {measured-BIA }}{ }^{\mathrm{a}}, \mathrm{kcal} / \mathrm{day} \\
\text { Correlation }^{b}, r\end{array}$ & $\begin{array}{l}1,573 \pm 220 \\
-11 \pm 162 \\
0.20^{*}\end{array}$ & $\begin{array}{l}1,619 \pm 274 \\
-41 \pm 258^{* *} \\
0.26^{* *}\end{array}$ & $\begin{array}{l}- \\
- \\
-\end{array}$ & $\begin{array}{l}- \\
- \\
-\end{array}$ \\
\hline
\end{tabular}

aBias was calculated as result obtained from reference method (MRI, DXA) minus BIA measurement. $95 \%$ limits of agreement was calculated as $\pm 2 \mathrm{SD}$.

${ }^{\mathrm{b}}$ Correlation was calculated as Pearson correlation coefficient for the relationship between $\left(\left(\right.\right.$ result reference method + result $\left.\left._{\mathrm{BIA}}\right) / 2\right)$ and the bias. $* \mathrm{p}<0.05$,

$* * \mathrm{p}<0.01$.

$* * * \mathrm{p}<0.001$.

${ }^{\dagger}$ Significant difference between BIA results and results from reference method by paired samples $\mathrm{t}$-test $(\mathrm{p}<0.01)$.
(LEXI, Tokyo, Japan). Briefly, the software employed knowledge-based image processing to label pixels as fat and nonfat components using a threshold for SM on the basis of the gray-level histograms of the images. Each slice was manually reviewed and voxels arising from nonmuscle compartments were deleted. Total volume of SM was determined from the sum of all SM areas $\left(\mathrm{cm}^{2}\right)$ multiplied by the slice thickness. SM $(\mathrm{kg})$ was calculated using a density for SM of $1.04 \mathrm{~g} / \mathrm{cm}^{3}[19]$. VF area was analyzed at the level L4-L5. Voxels arising from fatty bowel content were deleted. $\mathrm{VF}$ in $\mathrm{cm}^{2}$ was divided by 10 and rounded to derive $\mathrm{VF}_{\text {level }}$.

\section{Resting Energy Expenditure}

Indirect calorimetry was performed by using a ventilated hood system (Vmax Spectra 29n; SensorMedics BV, Bilthoven, Netherlands; software Vmax, version 12-1A) in the morning between 7.30 and 9.00 a.m. after an overnight fast. A detailed description of the method and its precision has been reported previously [20,21]. Briefly, the minimum duration of measurement was $30 \mathrm{~min}$. Before each measurement, calibration of flow and gas analyzers was performed. $\mathrm{VO}_{2}$ and $\mathrm{VCO}_{2}$ were converted to REE (kcal/24 h) using the abbreviated equation of Weir [22]. CVintra for between-day repeated measurements of REE was 5.0\% [20].

\section{Statistics}

Statistical analysis was performed using SPSS statistical software (SPSS 13.0, Inc., Chicago, IL, USA). All data are normally distributed and given as means \pm standard deviation (SD). Differences between men and women were analyzed by unpaired t-test. Differences between parameters of body composition assessed by different methods were tested by paired samples t-test. Analysis according to Bland and Altman was used to determine absolute agreement between the body composition variables assessed by criterion methods (MRI and DXA) and BIA as well as between REE measured by indirect calorimetry and predicted from BIA results
[23]. Linear regression analysis was used to determine the relative agreement between different methods. Pearson's correlation coefficient was calculated for relationships between variables. A $p$ value $<0.05$ was considered significant.

\section{Results}

Descriptive characteristics of the study population are summarized in table 1. Age ranged between 22 and 78 years. Four subjects were underweight (BMI 16.8-18.4 kg/m²), 47 were normal weight (BMI 18.5 to $<25.0 \mathrm{~kg} / \mathrm{m}^{2}$ ), 40 were overweight (BMI 25.0 to $<30 \mathrm{~kg} / \mathrm{m}^{2}$ ), and 16 were obese (BMI $\geq 30.0$ to $40.1 \mathrm{~kg} / \mathrm{m}^{2}$ ). When compared with men, women had a higher $\% \mathrm{FM}$ and a $\mathrm{VF}_{\text {level }}$ at concomitantly lower SM and REE. In table 2 correlation coefficients between parameters of body composition assessed by different BIA devices and reference methods are shown. \%FM and SM or TBBLM, determined by both Omron scales and the Tanita instrument, were highly related to that determined by DXA or MRI ( $\mathrm{r}$ values between 0.92 and $0.96, \mathrm{p}<0.001$ ). The correlation coefficients for $\% \mathrm{FM}$ and SM estimated by the Soehnle instrument were slightly lower $(\mathrm{r}=0.82$ and 0.84 ; table 2$)$. Although all BIA results displayed good absolute agreement with DXA and MRI for the assessment of either \%FM or SM (table 3, fig. 1,2), mean bias was significant for all BIA instruments $(\mathrm{p}<0.001)$, except for 

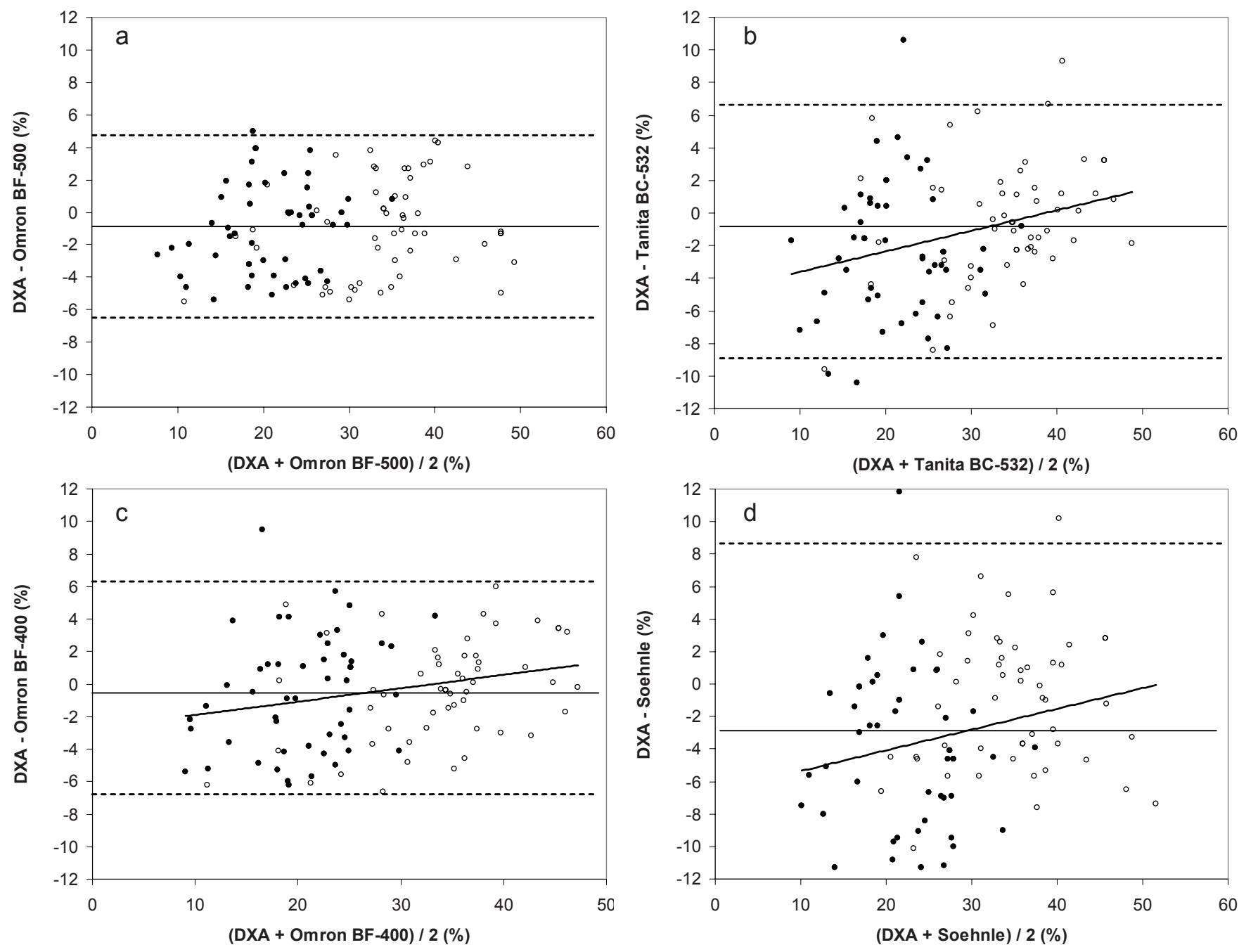

Fig. 1. Bland-Altman plots of limits of agreement in percent fat mass between DXA and a Omron BF-500, b Tanita, c Omron BF-400 and d Soehnle. Parallel lines indicate the mean difference \pm 2 SD. Open symbols for females, closed for males.

Omron BF-400 (\%FM) and Omron BF-500 (SM). The limits of agreement (mean bias and $95 \%$ confidence interval) were narrow for Omron BF-500 (-6.59 to $4.61 \% \mathrm{FM}$ and -4.62 to $4.74 \mathrm{~kg} \mathrm{SM})$ and wider for the other scales, $(-9.33$ to 6.59 \%FM and -7.43 to $5.85 \mathrm{~kg}$ TBBLM for Tanita BC-532; -7.1 to $6.14 \%$ FM for Omron BF-400; -14.54 to $8.58 \%$ FM and -9.52 to $3.92 \mathrm{~kg} \mathrm{SM}$ for Soehnle).

Systematic errors were observed for the assessment of i) \%FM using Tanita BC-532, Soehnle and Omron BF-400 instruments, and ii) SM using Omron BF-500. BIA overestimated \%FM at a low \%FM and underestimated it at a high \%FM (table 3, fig. 1). In addition, BIA tended to underestimate SM with increasing SM (table 3, fig. 2). The bias between \%FM assessed by DXA and BIA was inversely related to age ( $\mathrm{r}=-0.23, \mathrm{p}<0.05$ for Tanita, $\mathrm{r}=-0.52, \mathrm{p}<0.0001$ for Soehnle and $\mathrm{r}=-0.20, \mathrm{p}<0.05$ for Omron BF-400), height $(\mathrm{r}=-0.32$, $\mathrm{p}<0.001$ for Tanita) and positively associated with weight $(\mathrm{r}=0.22, \mathrm{p}<0.05$ for Omron BF-400). The bias between SM assessed by MRI and Soehnle instrument was positively related to age and height $(\mathrm{r}=0.32$ and $\mathrm{r}=0.24, \mathrm{p}<0.01)$, and negatively associated with BMI $(\mathrm{r}=-0.32, \mathrm{p}<0.001)$. The bias between TBBLM assessed by DXA and Tanita scale also correlated with age $(\mathrm{r}=0.25, \mathrm{p}<0.05)$. There was no systematic error in the assessment of \%FM by using Omron BF-500 compared with DXA and in the assessment of SM by using the Soehnle instrument compared with MRI.

Although both Omron BF-500 and Tanita BC-532 showed small and nonsignificant bias for $\mathrm{VF}_{\text {level }}$, the limits of agreement between $\mathrm{VF}_{\text {level }}$ assessed by BIA and MRI were wide, and a systematic bias was observed for Omron BF-500 (table 3). By contrast, both BIA instruments provided a good accuracy in the prediction of REE when compared with measured REE. 

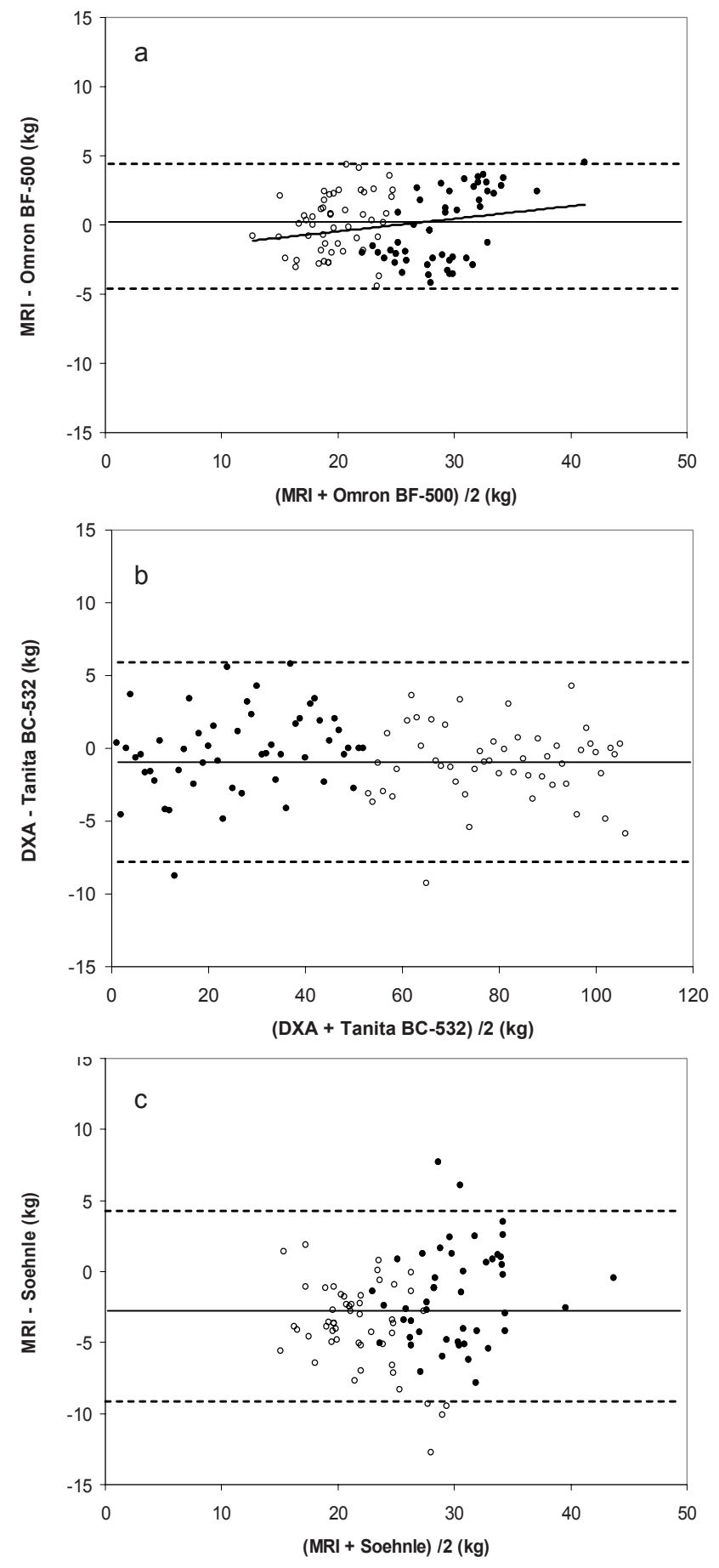

Fig. 2. Bland-Altman plots of limits of agreement in skeletal muscle mass or TBBLM $(\mathrm{kg})$ between MRI or DXA and a Omron BF-500, b Tanita BC-532 and $\mathbf{c}$ Soehnle. Parallel lines indicate the mean difference \pm 2 SD. Open symbols for females, closed for males

\section{Discussion}

Our study has shown a good relative agreement with DXA and MRI for all but one of the BIA consumer devices tested (table 2). Despite a small mean bias, the limits of agreement were considerably wide in all three foot-to-foot BIA devices (table 3). This result emphasizes the accurate application of bipolar BIA for estimation of body composition in groups, but also reveals its limited accuracy for the assessment of an individual. Inaccuracy of foot-to-foot BIA at the individual level has also been found by others. In a study by Xie et al. [24] the analysis of $95 \%$ limits of agreement showed that for most individuals \%FM estimated by foot-to-foot BIA differed from that measured by DXA by $12 \%$ below to $45 \%$ above. Mitsui et al. [25] reported a systematic bias with foot-to-foot BIA overestimating \%FM in lean subjects and underestimating $\% \mathrm{FM}$ in obese subjects, with only $45 \%$ of female and $47 \%$ of male subjects being accurately measured, i.e. within $10 \%$ of the measurement by DXA. In our study we can confirm the bias introduced by increasing \%FM. All foot-to-foot BIA devices overestimated $\% \mathrm{FM}$ at a low $\% \mathrm{FM}$ and underestimated it at a high \%FM (table 3, fig. 1). By contrast no systematic bias for \%FM was found using the tetrapolar BIA instrument. The narrowest limits of agreement were observed for the tetrapolar eight-electrode BIA device using eight tactile electrodes (table 3 ). This result is in line with previous publications comparing limits of agreement with DXA between footto-foot and hand-to-foot BIA devices $[14,26]$. In the study by Lazzer et al. [26], limits of agreement between DXA and two foot-to-foot BIA devices were much greater than those obtained with hand-foot BIA $(-7.7$ and $+4.3,-12.0$ and +10.6 vs. -2.1 and $+6.7 \mathrm{~kg}$ ). The authors concluded that foot-to-foot BIA could be acceptable to assess body composition in large groups of subjects, but cannot be recommended for body composition assessment in individuals because of the large errors in estimates.

Comparing four-tactile to eight-tactile electrodes and singleto multi-frequency BIA, \%FM was also more accurately estimated using eight electrodes and multifrequency electric current [14]. However, others have shown contradictory findings about the validity of multifrequency BIA. In overweight or obese men, multifrequency BIA when compared with DXA produced larger bias and wider limits of agreement than single-frequency BIA, and its accuracy estimating body composition was poor [27]. During weight loss in overweight young women, both multi- and single-frequency BIA showed small and similar biases ( 0.22 and $0.25 \% \mathrm{FM})$ and narrow limits of agreement $( \pm 3.4 \%$ for both) using DXA as a reference.

Thus multifrequency BIA at fixed frequencies may confer no advantage over single $50 \mathrm{kHz}$ BIA, whereas the additional use of hand electrodes may allow a more accurate prediction of body composition, reducing the prediction error in an individual case.

The accuracy of eight-electrode BIA (Tanita BC-418 equipment) has been shown to be negatively affected by abdominal obesity in women [28]. When compared with DXA, the underestimation of total and trunk FM by BIA significantly rose with increasing adiposity. However, we did not observe a significant correlation between the deviation of \%FM assessed by eight-electrode BIA and DXA and \%FM (table 3, fig. 1) trunk fat or waist circumference (data not shown). 
When compared to the measured $\mathrm{VF}_{\text {level }}$ the prediction of $\mathrm{VF}_{\text {level }}$ by both BIA devices was rather poor, with large limits of agreement (table 3). Thus bipolar or tetrapolar consumer devices using standard electrode placement provide only a qualitative index of VF. Newly developed BIA approaches using nonstandard electrode placement at the trunk seem to be more promising in estimation of VF [29].

In conclusion, BIA has a high potential as a accurate method of body composition analysis in public use. To meet these requirement, population-specific equations are needed. Therefore manufacturers should provide a characterization (age, height, weight, BMI, and ethnicity) of the reference population used to generate the algorithm. In addition, the reference method should be stated in the manual. For example, in the case of SM estimation by Tanita BC-532, TBBLM $M_{\text {DXA }}$ was used as a reference which differs considerably from SMMRI (table 1). Because of the lack of agreement between foot-tofoot BIA and DXA or MRI for the assessment of body composition in individuals, tetrapolar electrode arrangement is to be preferred for individual or public use. Bipolar devices pro- vide accurate results for field studies. The prediction of visceral fat by foot-to-foot as well as hand-foot BIA consumer devices provides only a qualitative index that may be useful to motivate subjects to lose weight.

\section{Acknowledgement}

The study was financed by a grant from Omron Healthcare Co, Kyoto, Japan.

Authors' contribution: study design (MJM, ABW), recruitment of subjects, data collection (ABW, WL, BH), data analysis (ABW), segmentation of MRI images (TS), discussion of results (MJM, ABW, EK, CCG, $\mathrm{MH}$ ), writing of the manuscript (ABW, MJM), MRI protocol (EK, CCG, $\mathrm{MH})$.

\section{Conflict of Interest}

TS is employed by Omron Healthcare Co, Kyoto, Japan, who financed the study.

\section{References}

1 Thompson R, Brinkworth GD, Buckley JD, Noakes M, Clifton PM: Good agreement between bioelectrical impedance and dual-energy X-ray absorptiometry for estimating changes in body composition during weight loss in overweight young women. Clin Nutr 2007;26:771-777.

2 Jebb SA, Siervo M, Murgatroyd PR, Evans S, Fruhbeck G, Prentice AM: Validity of leg-to-leg bioimpedance to estimate changes in body fat during weight loss and regain in overweight women: a comparison with multicompartment models. Int J Obes 2007:31:756-762.

3 Andreoli A, Melchiorri G, De Lorenzo A, Caruso I, Sinibaldi Salimei P, Guerrisi M: Bioelectrical impedance measures in different position and vs. dualenergy X-ray absorptiometry (DXA). J Sports Med Phys Fitness 2002;42:186-189.

4 Utter AC, Nieman DC, Ward AN, Butterworth DE: Use of the leg-to-leg bioelectrical impedance method in assessing body composition change in obese women. Am J Clin Nutr 1999;69:603-607.

5 Goldfield GS, Cloutier P, Mallory R, Prud'homme D, Parker T, Doucet E: Validity of foot-to-foot bioelectrical impedance analysis in overweight and obese children and parents. J Sports Med Phys Fitness 2006:46:447-453.

6 Frisard MI, Greenway FL, Delany JP: Comparison of methods to assess body composition changes during a period of weight loss. Obes Res 2005;13: 845-854.

7 Deurenberg P: Limitations of the bioelectrical impedance method for the assessment of body fat in severe obesity. Am J Clin Nutr 1996;64(3 suppl): $449 \mathrm{~S}-452 \mathrm{~S}$.

8 Segal KR, Van Loan M, Fitzgerald PI, Hodgdon JA Van Itallie TB: Lean body mass estimation by bioelectrical impedance analysis: a four-site cross-validation study. Am J Clin Nutr 1988;47:7-14.

9 Piers LS, Soares MJ, Frandsen SL, O'Dea K: Indirect estimates of body composition are useful for groups but unreliable in individuals. Int J Obes 2000;24:1145-1152.
10 Guo SS, Chumlea WC, Cockram DB: Use of statistical methods to estimate body composition. Am J Clin Nutr 1996;64(3 suppl):428S-435S.

11 Deurenberg-Yap M, Deurenberg P: Is a re-evaluation of WHO body mass index cut-off values needed? The case of Asians in Singapore. Nutr Rev 2003;61:S80-S87.

12 Sun SS, Chumlea WC, Heymsfield SB, Lukaski HC, Schoeller D, Friedl K, et al: Development of bioelectrical impedance analysis prediction equations for body composition with the use of a multicomponent model for use in epidemiologic surveys. Am J Clin Nutr 2003;77:331-340.

13 Rush EC, Chandu V, Plank LD: Prediction of fatfree mass by bioimpedance analysis in migrant Asian Indian men and women: a cross validation study. Int J Obes 2006;30:1125-1131.

14 Demura S, Sato S, Kitabayashi T: Percentage of total body fat as estimated by three automatic bioelectrical impedance analyzers. J Physiol Anthropol Appl Human Sci 2004;23:93-99.

15 Buchholz AC, Bartok C, Schoeller DA: The validity of bioelectrical impedance models in clinical populations. Nutr Clin Pract 2004;19:433-446.

16 Cornish BH, Ward LC, Thomas BJ, Jebb SA, Elia $\mathrm{M}$ : Evaluation of multiple frequency bioelectrical impedance and Cole-Cole analysis for the assessment of body water volumes in healthy humans. Eur J Clin Nutr 1996;50:159-164.

17 Chamney PW, Wabel P, Moissl UM, Müller MJ, Bosy-Westphal A, Korth O, Fuller NJ: A wholebody model to distinguish excess fluid from the hydration of major body tissues. Am J Clin Nutr 2007; 85:80-89.

18 Ellis KJ: Selected body composition methods can be used in field studies. J Nutr 2001;131:1589S$1595 \mathrm{~S}$

19 Synder WS, et al. Report of the Task Group on Reference Man. Oxford, Pergamon, 1975.
20 Bader N, Bosy-Westphal A, Dilba B, Müller MJ: Intra- and interindividual variability of resting energy expenditure in healthy male subjects - biological and methodological variability of resting energy expenditure. Br J Nutr 2005;94:843-849.

21 Korth O, Bosy-Westphal A, Zschoche P, Glüer CC, Heller M, Müller MJ: Influence of methods used in body composition analysis on the prediction of resting energy expenditure. Eur J Clin Nutr 2007; 61:582-589.

22 Weir JB: New methods for calculating metabolic rate with special reference to protein metabolism. J Physiol 1949;109:1-9.

23 Bland JM, Altman DG: Statistical methods for assessing agreement between two methods of clinical measurement. Lancet 1986;i:301-310.

24 Xie X, Kolthoff N, Bärenholt O, Nielsen SP: Validation of a leg-to-leg bioimpedance analysis system in assessing body composition in postmenopausal women. Int J Obes 1999;23:1079-1084.

25 Mitsui T, Shimaoka K, Tsuzuku S, Kajioka T, Sakakibara H: Accuracy of body fat assessment by bioelectrical impedance in Japanese middle-aged and older people. J Nutr Sci Vitaminol (Tokyo) 2006;52:154-156.

26 Lazzer S, Boirie Y, Meyer M, Vermorel M: Evaluation of two foot-to-foot bioelectrical impedance analysers to assess body composition in overweight and obese adolescents. Br J Nutr 2003;90:987-992.

27 Pateyjohns IR, Brinkworth GD, Buckley JD, Noakes M, Clifton PM: Comparison of three bioelectrical impedance methods with DXA in overweight and obese men. Obesity 2006;14:2064-2070.

28 Neovius M, Hemmingsson E, Freyschuss B, Uddén $\mathrm{J}$ : Bioelectrical impedance underestimates total and truncal fatness in abdominally obese women. Obesity 2006;14:1731-1738.

29 Nagai M, Komiya H, Mori Y, Ohta T, Kasahara Y, Ikeda Y: Development of a new method for estimating visceral fat area with multi-frequency bioelectrical impedance.Tohoku J Exp Med 2008;214: 105-112. 